\title{
The Improved $\exp (-\phi(\xi))$ Fractional Expansion Method and its Application to Nonlinear Fractional Sharma-Tasso-Olver Equation
}

\section{Alhakim LA and Moussa AA*}

Department of Management Information System and Production Management College of Business and Economics, Qassim University, Saudi Arabia

\begin{abstract}
In this paper, we propose a new method called $\exp (-\phi(\xi))$ fractional expansion method to seek traveling wave solutions of the nonlinear fractional Sharma-Tasso-Olver equation. The result reveals that the method together with the new fractional ordinary differential equation is a very influential and effective tool for solving nonlinear fractional partial differential equations in mathematical physics and engineering. The obtained solutions have been articulated by the hyperbolic functions, trigonometric functions and rational functions with arbitrary constants.
\end{abstract}

Keywords: Improved $\exp (-\phi(\xi))$ fractional expansion method; Nonlinear fractional Sharma-Tasso-Olver equation

\section{Introduction}

It is well known that nonlinear fractional partial differential equations (NFPDEs) are widely used as models to describe many important complex physical phenomena in various fields of science, such as plasma physics, nonlinear optics, solid state physics, fluid mechanics, fluid flow, chemical kinematics, chemistry, biology, finance, economy, and so on. Thus, establishing exact traveling wave solutions of NFPDEs is very important to better understand nonlinear phenomena's as well as other real-life applications.

In the past, a wide range of methods have been developed to generate analytical solutions of nonlinear partial differential equations. Among these methods are the $\left(\frac{G^{\prime}}{G}\right)$ expansion method $[1,2]$, the $\left(\frac{G^{\prime}}{G}, \frac{1}{G}\right)$ expansion method [3], the generalized of $\exp (-\phi(\xi)$ expansion method $[4,5]$,the coth a $(\xi)$ expansion method [6], the F-expansion method [7], and various other methods [8-11].

In recent years, several attempts have succeeded in the synthesis of the previous methods to searching for exact solutions to nonlinear fractional differential equations. Zhang and Zhang [12-14] proposed on the basis of homogeneous balance principle and Jumarie's modified Riemann-Liouville derivative a new direct method called fractional sub-equation method to search for explicit solutions of nonlinear time fractional biological population model and $(4+1)$ dimensional space-time fractional Fokas equation. Wangi and $\mathrm{Xu}$ [15] improved this method to obtain the exact solutions of the space-time fractional generalized Hirota-Satsuma coupled Korteweg-de Vries equations.

In this paper, we propose the improved exp $(-\phi(\xi))$ fractional expansion method for obtaining novel and more general exact traveling wave solutions for the nonlinear fractional Sharma TassoOlver equation $[12,13]$ :

$$
D_{t}^{\alpha} u+3 \delta u^{2} D_{x}^{\alpha} u+3 \delta\left(D_{x}^{\alpha} u\right)^{2}+3 \delta u D_{x}^{2 \alpha} u+\delta D_{x}^{3 \alpha} u=0 .
$$

where $0<\mathrm{a} \leq 1, \mathrm{u}=\mathrm{u}(\mathrm{x}, \mathrm{t}), \mathrm{t}>0, \delta$ is constant.

The remainder of the paper is organized as follows. Section 2 gives some definitions and properties of the modified RiemannLiouville derivative [16], and explains the improved $\exp (-\phi(\xi))$ fractional expansion method. Section 3 applies this method for solving the nonlinear fractional Sharma-Tasso-Olver equation. Section 4 concludes the paper.

\section{Jumarie's Modified Riemann-Liouville Derivative and the Improved $\exp (-\phi(\xi))$ Fractional Expansion Method}

In this section, we briefly review the main definitions and properties of the fractional calculus proposed by Jumarie [17] which will be used in the following section.

The modified Riemann-Liouville derivative as defined by Jumarie [18] is:

$$
D_{t}^{\alpha} f(\mathrm{t})=\left\{\begin{array}{l}
\left.\frac{1}{\Gamma(1-\alpha)} \frac{d}{d t} \int_{0}^{t}(\mathrm{t}-\xi)-f(0)\right) \mathrm{d} \xi, 0<\alpha \leq 1 \\
{\left[f^{[n]}(t)^{(\alpha-n)}, n \leq \alpha<n+1, n \geq 1\right]}
\end{array}\right.
$$

Some useful formulas and properties of Jumarie's modified Riemann-Liouville derivative were summarized in [18], among them the three following formulas:

$$
\begin{aligned}
& D_{t}^{\alpha} t^{r}=\frac{\Gamma(1+r)}{\Gamma(1+r-\alpha)} t^{r-\alpha}, r>0 \\
& D_{t}^{\alpha} f[f(t) g(t)]=g(t) D_{t}^{\alpha} f(\mathrm{t}) D_{t}^{\alpha} g(\mathrm{t}) \\
& D_{t}^{\alpha} f(g(\mathrm{t}))=\int_{g}^{t}[g(\mathrm{t})] D_{t}^{\alpha} f\left(g(\mathrm{t})=D_{t}^{\alpha} f\left(g(\mathrm{t})\left(g_{t}^{\prime \prime}(t)\right)^{\alpha}\right.\right.
\end{aligned}
$$

Now, we outline the main steps of the $\exp (-\phi(\xi))$ fractional expansion method to solve fractional differential equations. Suppose that a fractional partial differential equation, say in the independent variables $\mathrm{x}$ and $\mathrm{t}$, is given by

$$
F\left(u, u_{t}, u_{x}, D_{x}^{\alpha} u\right)=0,0<\alpha \leq 1
$$

Where $\mathrm{u}=\mathrm{u}(\mathrm{x}, \mathrm{t})$ is an unknown function, $\mathrm{F}$ is a polynomial in

*Corresponding author: Alaaeddin Amin Moussa, Department of Management Information System and Production Management College of Business and Economics, Qassim University, Buraidah, K.S.A, Saudi Arabia, Tel:+966 16380 0050; E-mail: Alaamath81@gmail.com

Received June 02, 2017; Accepted July 25, 2017; Published July 30, 2017

Citation: Alhakim LA, Moussa AA (2017) The Improved exp(- $\phi(\xi))$ Fractional Expansion Method and its Application to Nonlinear Fractional Sharma-Tasso-Olver Equation. J Appl Computat Math 6: 360. doi: 10.4172/2168-9679.1000360

Copyright: (c) 2017 Alhakim LA, et al. This is an open-access article distributed under the terms of the Creative Commons Attribution License, which permits unrestricted use, distribution, and reproduction in any medium, provided the original author and source are credited. 
$\mathrm{u}=\mathrm{u}(\mathrm{x}, \mathrm{t})$ and their various partial derivatives including fractional derivatives, $D_{t}^{\alpha} u$ and $D_{x}^{\alpha} u$ are the modified Riemann Liouville derivatives of $u$ with respect to $t$ and $x$, respectively. The main steps of this method are as follows:

Step 1: Use the traveling wave transformation:

$$
u(\mathrm{x}, \mathrm{t})=\mathrm{u}(\xi), \xi=x+k t
$$

Where $\mathrm{k}$ is a non-zero constant to be determined latter, which reduces (2.5) to an (NFODE) for $\mathrm{u}=\mathrm{u}(\xi)$ in the form:

$$
P\left(u, u_{\xi}, \mathrm{k}_{u \xi}, D_{\xi}^{\alpha} u, k^{\alpha}, D_{\xi}^{\alpha} u, \ldots\right)=0,0<\alpha \leq 1
$$

Step 2: Balance the highest derivative term with the nonlinear terms in (2.7) to find the value of the positive integer $(\mathrm{m})$. If the value $(\mathrm{m})$ is non-integer one can transform the equation studied.

Step 3: Suppose that the solution of (2.7) can be expressed as follows:

$$
u(\xi)=\sum_{i=-m}^{-1} \alpha_{i}(\exp (-\phi(\xi)))^{i}+\alpha_{0}+\sum_{i=1}^{m} \alpha_{i}(\exp (-\phi(\xi)))^{i}
$$

Where, $\alpha_{\mathrm{i}}(\mathrm{i}=0,1, \ldots, \mathrm{m})$ are constants to be determined, such that $\alpha \mathrm{i} 0$ and $\phi(\xi)$ satisfies the following fractional differential equation:

$$
D_{\xi}^{\alpha} \phi(\xi)=\exp (-\phi(\xi))+\mu \exp (\phi(\xi))+\lambda
$$

eqn. (2.9) gives the following solutions:

Family 1: When $\mu \neq 0,\left(\lambda^{2}-4 \mu\right)>0$,

$$
\phi_{1}(\xi)=\operatorname{In}\left(\frac{-\sqrt{\left(\lambda^{2}-4 \mu\right)} \tanh \left(\frac{\sqrt{\left(\lambda^{2}-4 \mu\right)}}{2}\left(\frac{\xi^{\alpha}+c \Gamma(1+\alpha)}{\Gamma(1+\alpha)}\right)\right)-\lambda}{2 \mu}\right)
$$

Family 2: When $\mu \neq 0,\left(\lambda^{2}-4 \mu\right)<0$,

$$
\phi_{2}(\xi)=\operatorname{In}\left(\frac{-\sqrt{\left(4 \mu-\lambda^{2}\right)} \tanh \left(\frac{\sqrt{\left(4 \mu-\lambda^{2}\right)}}{2}\left(\frac{\xi^{\alpha}+c \Gamma(1+\alpha)}{\Gamma(1+\alpha)}\right)\right)-\lambda}{2 \mu}\right)
$$

Family 3: When $\mu=0, \lambda \neq 0,\left(\lambda^{2}-4 \mu\right)>0$,

$$
\phi_{3}(\xi)=\operatorname{In}\left(\frac{\lambda}{\exp \left(\lambda\left(\frac{\xi^{\alpha}+c \Gamma(1+\alpha)}{\Gamma(1+\alpha)}\right)-1\right.}\right)
$$

Family 4: When $\mu \neq 0, \lambda \neq 0,\left(\lambda^{2}-4 \mu\right)=0$,

$$
\phi_{4}(\xi)=\operatorname{In}\left(\frac{2 \lambda\left(\frac{\xi^{\alpha}+c \Gamma(1+\alpha)}{\Gamma(1+\alpha)}\right)+4}{\lambda^{2}\left(\frac{\xi^{\alpha}+c \Gamma(1+\alpha)}{\Gamma(1+\alpha)}\right)}\right)
$$

Family 5: When $\mu=0, \lambda=0,\left(\lambda^{2}-4 \mu\right)=0$,

$$
\phi_{5}(\xi)=\operatorname{In}\left(\frac{\xi^{\alpha}+c \Gamma(1+\alpha)}{\Gamma(1+\alpha)}\right)
$$

Step 4: Substituting (2.8) into (2.7) and using (2.9), and then setting all the cofficients of $(\exp (-\phi(\xi)))^{\mathrm{i}}$ of the resulting systems to zero, yields a system of algebraic equations for $\mathrm{k}, \lambda, \mu$ and $\alpha_{\mathrm{i}}(\mathrm{i}=-\mathrm{m}, \ldots, \mathrm{m})$.

Step 5: Suppose that the value of the constants $k, \lambda, \mu$ and $\alpha_{i}$ $(\mathrm{i}=-\mathrm{m}, \ldots, \mathrm{m})$ can be found by solving the algebraic equations which are obtained in Step 4. Since the general solutions of (2.9) have been well known, substituting $k, \lambda, \mu, \alpha_{i}$ and the solutions of (2.9) into (2.8), we obtain the exact solutions for eqn. (2.5)

Note that if $\alpha=1$, then eqn. (2.9) becomes $\phi(\xi)=\exp (-\phi(\xi))+\mu$ exp $(\phi(\xi))+\lambda$, which is the foundation of the known $\exp (-\phi(\xi))$ expansion method for solving partial differential equations (PDEs). Thus, the above described $\exp (-\phi(\xi))$ fractional expansion method is the extension of the $\exp (-\phi(\xi))$ method to fractional case.

\section{The Exact Solution for Nonlinear Fractional Sharma Tasso-Olver Equation}

In this section, we will apply the improved $\exp (-\phi(\xi))$ fractional expansion method to find the exact solutions of the nonlinear fractional Sharma-Tasso-Olver equation:

$$
D_{t}^{\alpha} u+3 \delta u^{2} D_{x}^{\alpha} u+3 \delta\left(D_{x}^{\alpha} u\right)^{2}+3 \delta u D_{x}^{2 \alpha} u+\delta D_{x}^{3 \alpha} u=0
$$

where $0<\alpha \leq 1$. In ref. [19], the authors solved eqn. (3.1) by a proposed fractional sub-equation method based on the fractional Riccati equation $D_{\xi}^{\alpha} \phi(\xi)=r+q \phi(\xi)+\mathrm{p} \phi(\xi)^{2}$.

Now we will apply the described method in Section 2 to eqns. (3.1). To do so, Suppose that

$$
\mathrm{u}(\mathrm{x}, \mathrm{t})=\mathrm{u}(\xi), \xi=\mathrm{x}+\mathrm{kt} \text {, }
$$

where $\mathrm{k}$ is a constant. Substituting (3.2) into eqn. (3.1), gives the following nonlinear fractional ordinary differential equations:

$$
k^{\alpha} D_{\xi}^{\alpha} u+3 \delta u^{2} D_{\xi}^{\alpha} u+3 \delta\left(D_{\xi}^{\alpha} u\right)^{2}+3 \delta u^{2} D_{\xi}^{2 \alpha} u+\delta D_{\xi}^{3 \alpha} u=0 .
$$

Suppose that eqn. (3.3) has the following solution:

$$
u(\xi)=\sum_{i=-m}^{-1} \alpha_{i}(\exp (-\phi(\xi)))^{i}+\alpha_{0}+\sum_{i=1}^{m} \alpha_{i}(\exp (-\phi(\xi)))^{i}
$$

where $\alpha_{\mathrm{i}}(\mathrm{i}=-\mathrm{m}, \ldots, \mathrm{m})$ are constants to be determined later. Balancing the order of $\mathrm{D}^{3 a}{ }_{\xi} \mathrm{u}$ and $\mathrm{u}^{2} \mathrm{D}^{\alpha}{ }_{\xi} \mathrm{u}$, we find $\mathrm{m}=1$. So,

$$
\mathrm{u}(\xi)=\alpha_{0}+\alpha 1 \exp (-\phi(\xi))+\alpha_{-1}(\exp (-\phi(\xi)))^{-1}
$$

Substituting eqns. (3.5) and (2.9) into eqn. (3.3), the left-hand side is converted into polynomials in $(\exp (-\phi(\xi)))^{ \pm j},(\mathrm{j}=0,1,2, \ldots$.$) . By collecting$ each coefficient of these resulted polynomials to zero, we obtain a set ofsimultaneous algebraic equations, which are not presented for sake of clarity, for $\alpha_{0}, \alpha_{1}, \alpha_{-1}, \lambda, \mu$ and $k$. Solving these algebraic equations with the help of algebraic software Maple, we obtain:

$$
\text { Case 1: } \alpha_{0}=0, \alpha_{1}=1, \alpha_{-1}=-\mu, \lambda=\lambda, \mu=\mu, \mathrm{k}=k=\exp \left(\frac{\operatorname{In}\left(-\delta\left(\lambda^{2}-4 \mu\right)\right.}{\alpha}\right)
$$

Substituting eqn. (3.6) into eqn. (3.5), we have:

$$
\mathrm{u}(\xi)=\exp (-\phi(\xi))-\mu \exp (\phi(\xi))
$$

Where $\xi=x+\exp \left(\frac{\operatorname{In}\left(-\delta\left(\lambda^{2}-4 \mu\right)\right.}{\alpha}\right) t$

Consequently, the exact solution of the of the nonlinear fractional Sharma-Tasso-Olver equation (3.1) with the help of eqn. (2.10) to eqn. (2.14), are obtained in the following form:

Case (1-1): When $\mu \neq 0,\left(\lambda^{2}-4 \mu\right)>0$, 


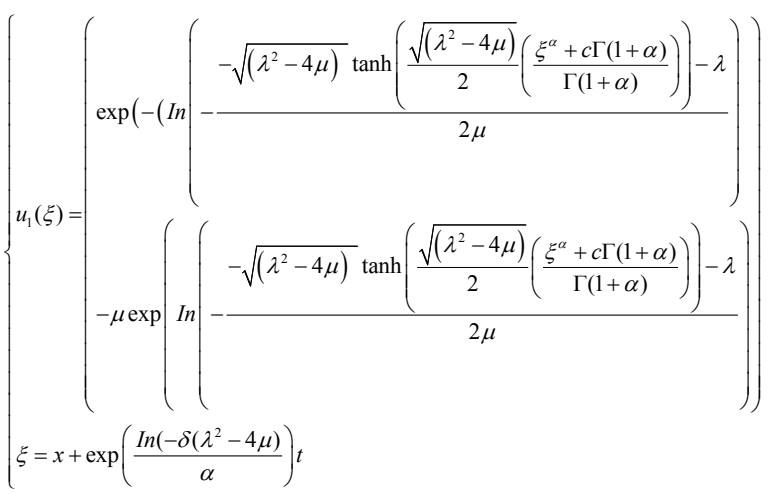

Case (1-2): When $\mu \neq 0,\left(\lambda^{2}-4 \mu\right)<0$,

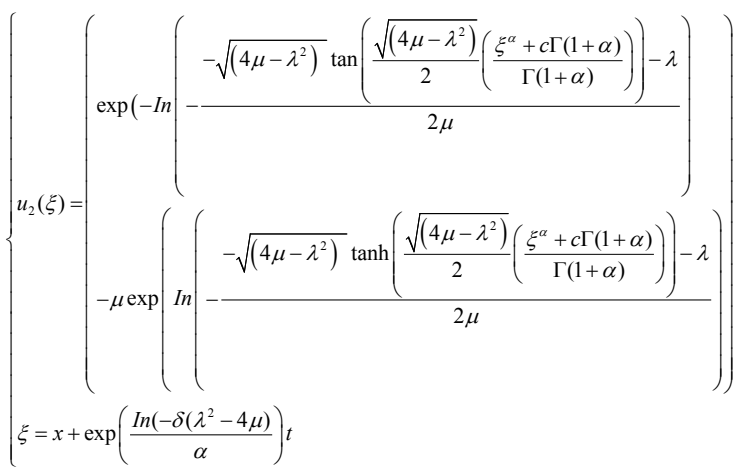

Case (1-3): When $\mu=0, \lambda \neq 0,\left(\lambda^{2}-4 \mu\right)>0$,

$$
\left\{u_{3}(\xi)=\exp \left(\begin{array}{l}
\operatorname{In}\left(\frac{\lambda}{\exp \left(\lambda\left(\frac{\xi^{\alpha}+c \Gamma(1+\alpha)}{\Gamma(1+\alpha)}\right)-1\right.}\right) \\
\xi=x+\exp \left(\frac{\operatorname{In}\left(-\delta \lambda^{2}\right)}{\alpha}\right) t
\end{array}\right)\right.
$$

Case (2): $\alpha_{0}=\alpha_{0}, \alpha_{1}=1, \alpha_{-1}=0, \lambda=\lambda, \mu=\mu, \mathrm{k}=k=\ln \left(\frac{\operatorname{In}\left(-\delta\left(3 \alpha_{0}^{2}-3 \alpha_{0}^{2} \lambda+\lambda^{2}-\mu\right)\right.}{\alpha}\right)$

Substituting (3.11) into (3.5), we have:

$\mathrm{u}(\xi)=\alpha_{0}+\exp (-\phi(\xi))$,

Where $\xi=x+\exp \left(\frac{\operatorname{In}\left(-\delta\left(3 \alpha_{0}^{2}-3 \alpha_{0}^{2} \lambda+\lambda^{2}-\mu\right)\right.}{\alpha}\right)$

Consequently, the exact solution of the of the nonlinear fractional Sharma-Tasso-Olver equation (3.1) with the help of eqn. (2.10) to eqn. (2.14), are obtained in the following form:

Case (2-1): When $\mu \neq 0,\left(\lambda^{2}-4 \mu\right)>0$,

$$
\left\{\begin{array}{l}
u_{4}(\xi)=\alpha_{0}+\exp \left(-\operatorname{In} \frac{\left(\left(\lambda^{2}-4 \mu\right) \tanh \left(\frac{\left.\sqrt{\left(\lambda^{2}-4 \mu\right.}\right)}{2}\left(\frac{\xi^{\alpha}+c \Gamma(1+\alpha)}{\Gamma(1+\alpha)}\right)-\lambda\right)\right.}{2 \mu}\right) \\
\xi=x+\exp \left(\frac{\operatorname{In}\left(-\delta\left(3 \alpha_{0}^{2}-3 \alpha_{0}^{2} \lambda+\lambda^{2}-\mu\right)\right.}{\alpha}\right) t
\end{array}\right.
$$

Case (2-2): When $\mu \neq 0,\left(\lambda^{2} 4 \mu\right)<0$

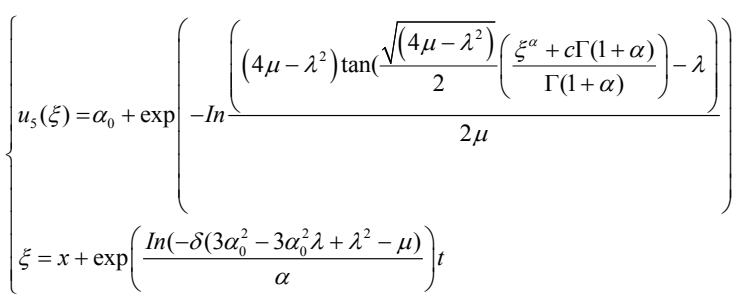

Case (2-3): When $\mu=0, \lambda \neq 0,\left(\lambda^{2}-4 \mu\right)>0$

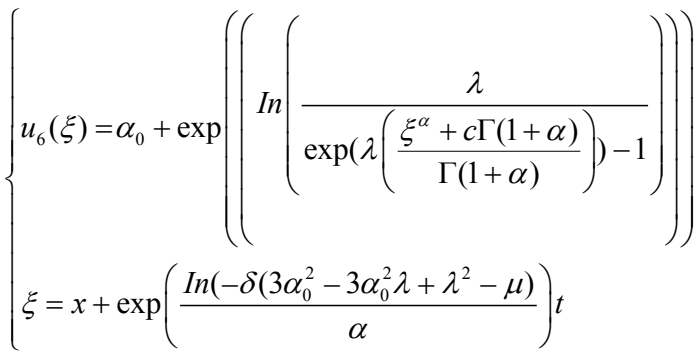

Case (2-4): When $\mu \neq 0, \lambda \neq 0,\left(\lambda^{2}-4 \mu\right)=0$,

$$
\left\{\begin{array}{l}
u_{7}(\xi)=\alpha_{0}+\exp \left(\left(-\operatorname{In}\left(\frac{2 \lambda\left(\frac{\xi^{\alpha}+c \Gamma(1+\alpha)}{\Gamma(1+\alpha)}\right)+4}{\lambda^{2}\left(\frac{\xi^{\alpha}+c \Gamma(1+\alpha)}{\Gamma(1+\alpha)}\right)}\right)\right)\right. \\
\xi=x+\exp \left(\frac{\operatorname{In}\left(-\delta\left(3 \alpha_{0}^{2}-3 \alpha_{0}^{2} \lambda+\lambda^{2}-\mu\right)\right.}{\alpha}\right) t
\end{array}\right.
$$

Case (2-5): When $\mu=0, \lambda=0,\left(\lambda^{2}-4 \mu\right)=0$,

$\left\{\begin{array}{l}u_{8}(\xi)=\alpha_{0}+\exp \left(\left(-\operatorname{In}\left(\frac{\xi^{\alpha}+c \Gamma(1+\alpha)}{\Gamma(1+\alpha)}\right)\right)\right) \\ \xi=x+\exp \left(\frac{\operatorname{In}\left(-3 \delta \alpha_{0}^{2}\right)}{\alpha}\right) t\end{array}\right.$

Case (3): $\alpha_{0}=\lambda, \alpha_{1}=2, \alpha_{-1}=0, \lambda=\lambda, \mu=\mu, k=\exp \left(\frac{\operatorname{In}\left(-\delta\left(\lambda^{2}-4 \mu\right)\right.}{\alpha}\right)$

Substituting (3.18) into (3.5), we have:

$\mathrm{U}(\xi)=\lambda+2 \exp (-\phi(\xi))$

Where $\xi=x+\exp \left(\frac{\operatorname{In}\left(-\delta\left(\lambda^{2}-4 \mu\right)\right.}{\alpha}\right)$.

Consequently, the exact solution of the of the nonlinear fractional Sharma-Tasso-Olver equation (3.1) with the help of eqn. (2.10) to eqn. (2.14), are obtained in the following form:

Case (3-1): When $\mu \neq 0,\left(\lambda^{2}-4 \mu\right)>0$,

$$
\left\{\begin{array}{l}
u_{9}(\xi)=\lambda+2 \exp \left(-\operatorname{In} \frac{\left(\lambda^{2}-4 \mu \tan \left(\frac{\sqrt{\left(\lambda^{2}-4 \mu\right)}}{2}\left(\frac{\xi^{\alpha}+c \Gamma(1+\alpha)}{\Gamma(1+\alpha)}\right)-\lambda\right)\right.}{2 \mu}\right) \\
\xi=x+\exp \left(\frac{\operatorname{In}\left(-\delta\left(\lambda^{2}-4 \mu\right)\right.}{\alpha}\right) t
\end{array}\right.
$$

Case (3-2): When $\mu \neq 0,\left(\lambda^{2}-4 \mu\right)<0$, 


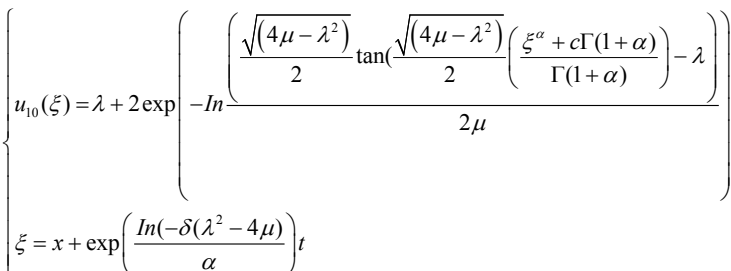

Case (3-3): When $\mu=0, \lambda \neq 0,\left(\lambda^{2}-4 \mu\right)>0$,

$$
\left\{\begin{array}{l}
\left.u_{11}(\xi)=\lambda+2 \exp \left(\mid-\operatorname{In}\left(\frac{\lambda}{\exp \left(\lambda\left(\frac{\xi^{\alpha}+c \Gamma(1+\alpha)}{\Gamma(1+\alpha)}\right)-1\right)}\right)\right)\right) \\
\xi=x+\exp \left(\frac{\operatorname{In}\left(-\delta \lambda^{2}\right)}{\alpha}\right) t
\end{array}\right.
$$

Case (4): $\alpha_{0}=\alpha_{0}, \alpha_{1}=0, \alpha_{-1}=-\mu, \lambda=\lambda, \mu=\mu, \mathrm{k}=\exp \left(\exp \frac{\operatorname{In}\left(\delta\left(\mu-\lambda^{2}-3 \alpha_{0}^{2}-3 \lambda \alpha_{0}\right)\right.}{\alpha}\right)(3.2$

Substituting (3.23) into (3.5), we have:

$\mathrm{u}(\xi)=\alpha_{0}-\mu(\exp (-\phi(\xi)))^{-1}$

Where $\exp \frac{\operatorname{In}\left(\delta\left(\mu-\lambda^{2}-3 \alpha_{0}^{2}-3 \lambda \alpha_{0}\right)\right.}{\alpha}$

Consequently, the exact solution of the of the nonlinear fractional Sharma-Tasso-Olver equation (1. 1) with the help of eqn. (2.10) to eqn. (2.14), are obtained in the following form:

Case (4-1): When $\mu /=0,(\lambda 2-4 \mu)>0$,

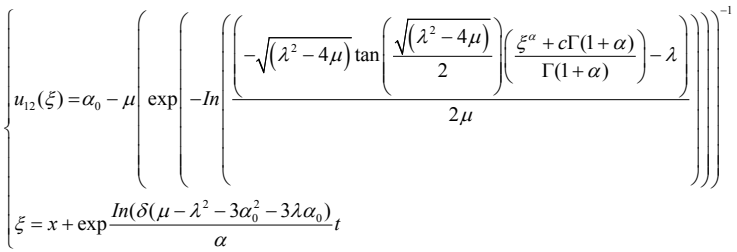

Case (4-2): When $\mu \neq 0,\left(\lambda^{2}-4 \mu\right)<0$,

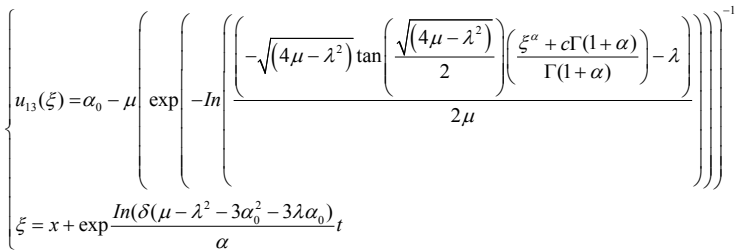

Case (4-3): When $\mu \neq 0, \lambda \neq 0,\left(\lambda^{2}-4 \mu\right)=0$,

$$
\left\{\begin{array}{l}
u_{14}(\xi)=\alpha_{0}-\mu\left(\exp \left(-\operatorname{In}\left(\frac{\left.2 \lambda\left(\frac{\xi^{\alpha}+c \Gamma(1+\alpha)}{\Gamma(1+\alpha)}\right)+4\right)}{\left(\lambda^{2}\left(\frac{\xi^{\alpha}+c \Gamma(1+\alpha)}{\Gamma(1+\alpha)}\right)\right)}\right)\right)\right)^{-1} \\
\xi=x+\exp \left(\frac{\operatorname{In}\left(-3 \delta\left(\frac{1}{4} \lambda^{2}+\alpha_{0}^{2}+\lambda \alpha_{0}\right)\right.}{\alpha}\right) t
\end{array}\right.
$$

Substituting (3.28) into (3.5), we have:

$\mathrm{U}(\xi)=\alpha_{0}-2 \mu(\exp (-\phi(\xi)))^{-1}$.
Where $\xi=x+\exp \left(\frac{\operatorname{In}\left(-\delta\left(\lambda^{2}-4 \mu\right)\right.}{\alpha}\right) t$

Consequently, the exact solution of the of the nonlinear fractional Sharma-Tasso-Olver equation (3.1) with the help of eqn. (2.10) to eqn. (2.14), are obtained in the following form

Case (5-1): When $\mu \neq 0,\left(\lambda^{2}-4 \mu\right)>0$,

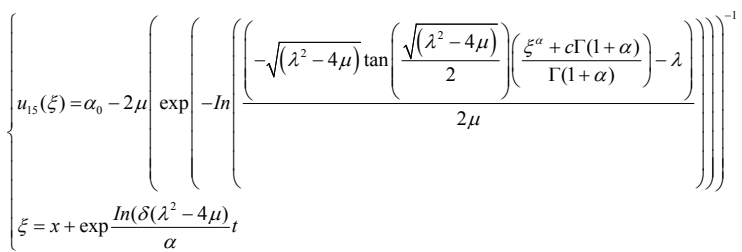

Case (5-2): When $\mu \neq 0,\left(\lambda^{2}-4 \mu\right)<0$,

$$
\left\{\begin{array}{l}
\left.\left.\left.u_{16}(\xi)=\alpha_{0}-2 \mu\left(\exp \left(\ln \left(\frac{\left(-\sqrt{\left(4 \mu-\lambda^{2}\right)} \tan \left(\frac{\sqrt{\left(4 \mu-\lambda^{2}\right)}}{2}\right)\left(\frac{\xi^{\alpha}+c \Gamma(1+\alpha)}{\Gamma(1+\alpha)}\right)-\lambda\right)}{2 \mu}\right)\right)\right)^{-1}\right)\right)\right)^{-1} \\
\xi=x+\exp \frac{\ln \left(\delta\left(\lambda^{2}-4 \mu\right)\right.}{\alpha} t
\end{array}\right.
$$

\section{Conclusion}

In this article, we proposed a new method called improved exp $(-\phi(\xi))$ fractional expansion method using the generalized wave transformation (2.6) and the auxiliary fractional differential equation (2.9), to obtain the exact solutions of nonlinear fractional SharmaTasso-Olver equation. The main advantage of this method is its capability of greatly reducing the size of computational work compared to existing techniques. The method could be used for a large class of very interesting nonlinear equations. These solutions have rich local structures, it may be important to explain some physical phenomena. This work shows that, the improved $\exp (-\phi(\xi))$ fractional expansion method is direct, effective and can be used for many other FNLPDEs in mathematical physics.

\section{References}

1. Wang $\mathrm{M}, \mathrm{X} \mathrm{Li}$, Zhang $\mathrm{J}(2008)$ The $\left(\frac{G^{\prime}}{G}\right)$ 'expansion method and travelling wave solutions of nonlinear evolution equations in mathematical physics. Physics Letters A 372: 417-423.

2. Bashir MA, Moussa AA(2014) New Approach of $\left(\frac{G^{\prime}}{G}\right)$ Expansion Method. Applications to K d V Equation. Journal of Mathematics Research 6: 24-31.

3. Li LX, Li Eq, Wang M (2010) The $\left(\frac{G^{\prime}}{G}, \frac{1}{G}\right)$ expansion method and its application to travelling wave solutions of the Zakharov equation, Applied Mathematics $B$ 25: 454-462.

4. Bashir MA, Moussa AA, Alhakim LA (2015) The new generalized of $\exp (-\phi(\xi))$ expansion method and its application to some complex nonlinear partia differential equation. Journal of Advanced in Mathematics 9: 2905-2926.

5. Yahya KH, Moussa ZA (2015) New approach of generalized $\exp (-\phi(\xi))$ expansion method and its application to some nonlinear partial differentia equation. Journal of Mathematics Research 7: 106-121.

6. Bashir MA , Moussa AA (2014) The cotha (६)Expansion Method and its Application to the Davey-Stewarts on Equation. Applied Mathematical Sciences 8: 3851-3868.

7. Bashir MA , Alhakim LA(2013) New F Expansion method and its applications to Modified KdV equation. Journal of Mathematics Research 5: 38-94. 
Citation: Alhakim LA, Moussa AA (2017) The Improved exp $(-\phi(\xi))$ Fractional Expansion Method and its Application to Nonlinear Fractional SharmaTasso-Olver Equation. J Appl Computat Math 6: 360. doi: 10.4172/2168-9679.1000360

8. Abdou MA (2007) The extended F-expansion method and its application for a class of nonlinearevolution equations. Chaos. Solitons and Fractals 31: 95-104.

9. Chen HT, Zhang HQ (2003) Improved Jacobin elliptic method and its applications. Chaos. Solitons and Fractals 15: 585-591

10. Mirzazadeh M, Khaleghizadeh S (2013) Modification of truncated expansion method to some complex nonlinear partial differntial equations. Acta Universitatis Apulensis 33: 109-116.

11. Liu SK, Fu ZT, Liu SD, Zhao Q (2001) Jacobi elliptic function expansion method and periodic wave solutions of non linear wave equations, Physics Letters $A$ $289: 69-74$.

12. Jafari $H$, Tajadodi $H$, Baleanu D, Al-Zahrani AA, Alhamed YA, et al., (2013) Fractional sub-equation method for the fractional generalized reacction During model andnonlinear fractional Sharma-Tasso-Olver equation.Cent. Eur. J. Phys 11: 1482-1486.

13. Zhang GY, Feng Q (2013) Fractional Riccati equation rational expansion method forfractional differential equations. Appl. Math. Inf. Scie 7: 1575-1584.
14. Zhang S, Zhang HQ (2011) Fractional sub-equation method and its applications to nonlinear fractional PDEs.Physics Letters A 375: 1069-1073.

15. Wangi GW, Xu TZ (2014) The modified fractional sub-equation method and its applications to nonlinear fractional partial differential equations, Oom. Journ. Phys 59: 636-645.

16. Jumarie G (2006) Modified Riemann-Liouville derivative and fractional taylor series of non differentiable functions further results. Computers and Mathematics with Applications 51: 1367-1376

17. Jumarie $G(2010)$ Cauchy's integral formula via the modified Riemann-Liouville derivative for analytic functions of fractional order. Applied Mathematics Letters 23: $1444-1450$.

18. Jumarie $G$ (2009) Table of some basic fractional calculus formulae de-rived from a modified Riemann-Lioville derivative for non-differentiable functions, Applied Mathematics Letters 22: 378-385.

19. G'omez SCA (2014) An improved sub-equation method for solving nonlinear fractional equations, International. Journal of Pure and Applied Mathematics 101: $133-140$ 